\title{
The Effects of Radiation Pressure on Orbiting Mirrors with Non-Zero Eccentricity
}

\author{
Hannah Bechtel
}

Physics, University of Wisconsin - La Crosse

\begin{abstract}
The search for extraterrestrial life has been approached from many angles. For Korpela, et al, among others, the search comes in the form of analyzing stars' light curves for hints of satellites around exoplanets. Such satellites could be in the form of large, thin, lightweight mirrors, possibly used to redirect sunlight from a star to the surface of a planet. The feasibility and fuel efficiency of such an undertaking would depend heavily on the effects of radiation pressure. My colleagues and I have simulated those satellites using REBOUND (Rein and Liu 2012), an N-body simulator, to understand the effect of radiation pressure on mirrors orbiting an exoplanet. We changed the types of stars, initial orbit orientations, eccentricities, and other variables. Each simulation ran to a pre-set 1000 orbits or until the mirror crashed into the planet or escaped orbit. A list of survival times and other data trends was compiled to identify potentially stable (or quasi-stable) orbits. Overall, it appeared that simulations with initial eccentricity close to zero $(0.1$, sometimes 0.3$)$ were more like previously investigated initially circular simulations. There were extreme exceptions to this however; some of the initially eccentric simulations lasted for 1000 orbits, while the equivalent initially circular simulations crashed or escaped very early on. We also used the data output from the simulations to create plots like a mirror's distance from the planet over time and a mirror's eccentricity over time in order to find possible stability trends. We are still investigating potentially stable configurations.
\end{abstract}

\section{Introduction:}

In 1995, they discovered a planet (51 Pegasus b) orbiting a star very like our sun. This opened our eyes to the possibility that there are Earth-like planets in our Galaxy and other galaxies. Since then we've discovered around 4,400 extrasolar planets ${ }^{1}$, or exoplanets. The most common way of detecting these planets is to use what's known as the transit method. When a planet passes in front of its star, it blocks a portion of that star's light. We can measure that difference in what's known as a transit light curve.

Only around 23 exoplanets are Earth-like and potentially habitable ${ }^{2}$. Others are tidally locked (one side faces the star at all times), or are gaseous giants, or their orbit is located in an uninhabitable region of space around their stars ${ }^{3}$. Astronomers are looking for exoplanets that are located in habitable zones (the region of space around a star that is both cool enough and warm enough to have liquid surface water on planets with atmospheres).

1

1 Re

2

${ }^{3}$ Retrieved from: https://www.livescience.com/earth-sized-planet-in-habitable-zone.html 
In 2015, Eric Korpela, Shauna Sallmen, and Diana Leystra Greene theorized that an unknown alien civilization might want to use orbiting mirrors to redirect sunlight to the dark side of a tidally locked exoplanet. They suggested that a "cloud" of mirrors would be visible in the transit light curve of an exoplanet's parent star. An obstacle to this kind of satellite constellation is radiation pressure, which is the pressure that photons from the star exert on an object due to the exchange of momentum between the photons and that object.

We would like to know exactly how radiation pressure affects orbits so we can identify relatively stable, fuel-efficient orbits. My research group is running simulations to test the stability of a single mirror orbiting an Earth-sized planet, with and without radiation pressure (RP). My colleague, Kaisa Crawford-Taylor, wrote a package of programs and scripts for running the simulations. This code uses Python 3 and REBOUND (Rein \& Liu 2012).

This paper highlights some of the results of over 1500 simulations with a large variety of initial mirror orbit sizes, shapes, and orientations around potentially habitable Earth-sized planets orbiting stars whose lifetimes allow for the possible development of synchronous planet rotation.

\section{Methods:}

Using the python language, the REBOUND packages, and the package that my colleague wrote, I ran over 1500 simulations with varying initial conditions until the mirrors crashed into the planet, escaped orbit, or for 1000 initial mirror orbit periods. These simulations involve six types of stars, M0, M5, M8, K0, K5, and G2, since the habitable planets of cool, low-mass stars are the most likely to have a dark side. The planets we simulate as orbiting these stars are Earth-sized and located at the inner edge of the habitable zone $\left(\mathrm{HZin}^{4}\right)$. These are the ones most likely to be tidally locked for all these star types, but potentially can still support liquid water.

The mirrors in our simulations are one square kilometer and $1000 \mathrm{~kg}$. They are in initially eccentric orbits (eccentricity $=0.1,0.3,0.5$, and 0.8 ) with orbit sizes of 2,3 , and 10 Earth radii. The eccentricity represents how elongated the orbit is; an orbit with an eccentricity of 0.1 is more similar to a circular orbit than if an orbit had eccentricity of 0.5 or 0.8 . The planet orbit around the star defines the $X Y$ plane for all simulations. There are four main initial orbit orientations for the mirrors: orbiting in the XY-plane, the XZ-plane, the YZ-plane, or going 'backwards' in the XYplane (-XY). Figure 1 shows these three mirror orbit orientations.

4Retrieved from: https://science.nasa.gov/science-pink/s3fs-public/atoms/files/nasa 2018 strategic plan_0.pdf 


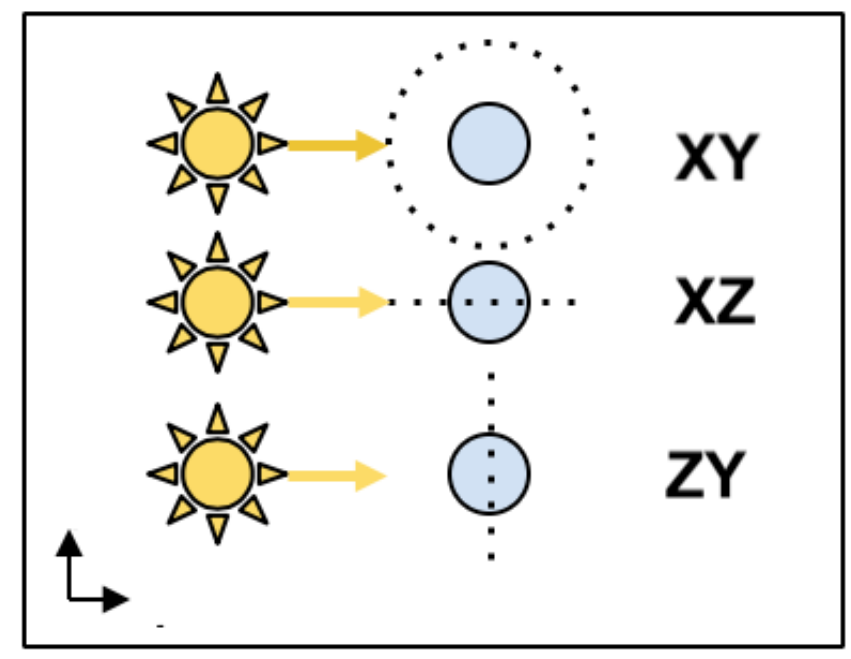

Figure 1: Schematic diagram showing the different options for initial mirror orbit orientations. The planet orbits the star in the XY plane. Source: Kaisa Crawford-Taylor

For all of the orientations (XY, $-\mathrm{XY}, \mathrm{XZ}, \mathrm{ZY})$, we simulated two initial secondary orientations that differ in whether the mirror is initially near to or far from the planet. Figure 2 shows the secondary orientations for the primary orientation of XY. We wanted the mirrors to all start on the side of the planet away from the star.
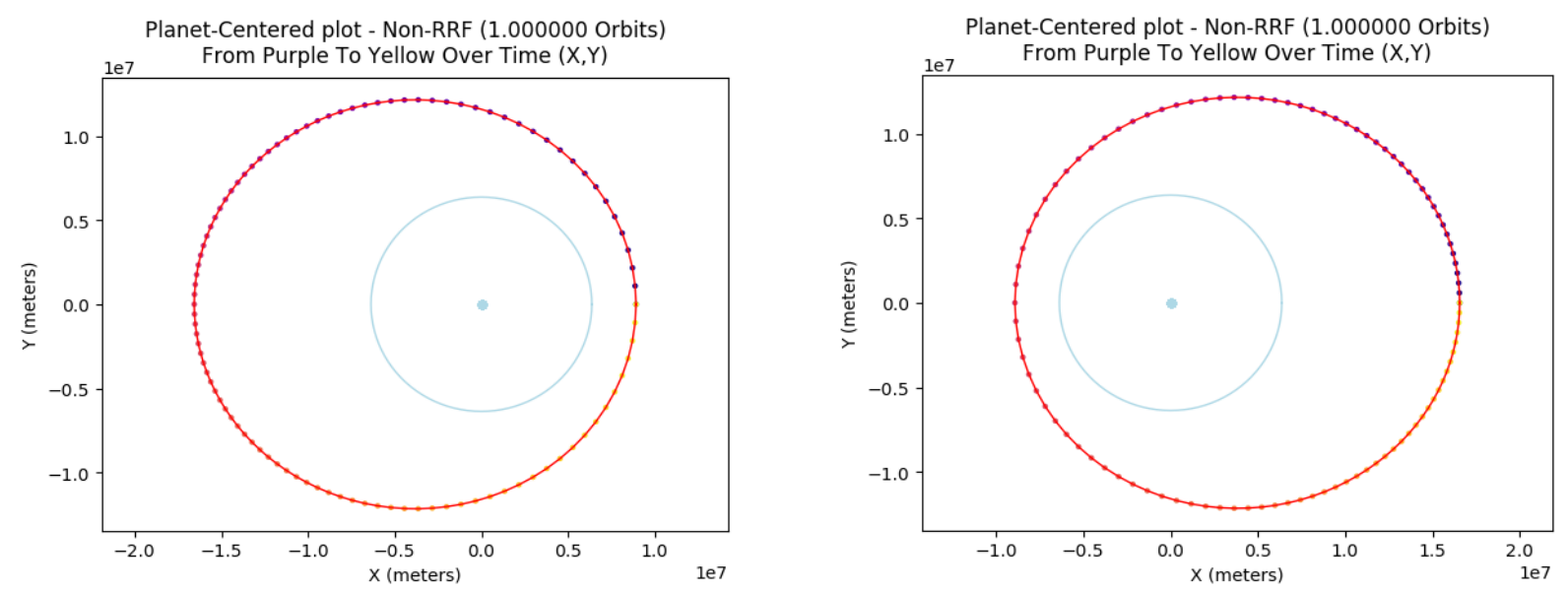

Figure 2: An XY simulation with secondary mirror orbit orientations of near (left) and far (right). In both simulations, the blue dot represents the center of mass of the planet, while the blue line represents the surface of the planet. The path the mirror traces out is shown by the connected dots, where it goes from purple to yellow over time.

Radiation pressure must be included in the simulations because photons from a star carry a nonzero momentum. When those photons are incident on the mirror, some of the momentum is transferred, essentially "pushing" it. At every point in the simulation, the acceleration due to RP was calculated using the following equation, where $L$ is stellar luminosity, $A$ is mirror area, $\theta$ is angle of incidence of starlight relative to the mirror's normal, $c$ is the speed of light, $m$ is mirror mass, and $d$ is planet location relative to the star. 


$$
a_{R P}=\frac{2 L A(\cos \theta)^{2}}{4 \pi c d m}
$$

We have two options for radiation pressure. For "Always RP", the radiation pressure affects the mirror all the time. For "Night RP", the radiation pressure only affects the mirror when it's on the dark side of the planet. In both cases, the mirrors redirect starlight towards the center of the planet; however, "Always RP" would heat the planet more than "Night RP". For comparison, we also run simulations without radiation pressure.

All of the simulations are run for either 1000 'mirror orbits' or until they touch the atmosphere or reach escape velocity. A 'mirror orbit' is the time it would take the mirror to go around the planet one time if there were no outside influences; i.e. no radiation pressure or stellar gravitational effects. These simulations use REBOUND's IAS15 integrator (Rein \& Spiegel 2014).

Each simulation recorded mirror orbital elements, as well as object positions and velocities at specified times throughout the simulation. It also created images showing the path of the mirror around the planet in various projections. After I ran the simulations, I compiled the results into a table and plots that allowed me to compare the elliptical orbits to each other and to the circular ones. I started by looking at simulation end state (survive, crash, or escape) and mirror survival times.

Then I explored mirror orbit evolution by examining how fast important orbital elements change and plotting the mirror's distance from and velocity relative to the planet throughout the simulation. To do this I wrote a python tool to plot the following orbital elements vs time for each simulation: eccentricity, semi-major axis, and period, as well as the change in each of those. I also wrote a decorator (a python function that can execute other functions) to plot all of these as well as the mirror's distance from the planet and the mirror's velocity for a list of simulations. This helped to analyze the data output by the package.

In summary, I obtained data for about 1500 simulations with varied initial eccentricities, orbit orientations, and mirror orbit radii. Each simulation recorded information about the mirror (see above) at specified times throughout the simulation and output graphical representations of its path. From the output data, I summarized the survival time and end state of each simulation. I also made plots of the mirror distance relative to the planet, the mirror's velocity compared to its escape velocity, the mirror's eccentricity, period, semi-major axis, and omega, as well as the change in those orbital elements.

\section{Results:}

In this section I summarize and highlight the results of my preliminary analysis of the simulations.

Plotting the mirror's distance relative to the planet usually allowed us to see at a quick glance if an orbit would be stable long term; if it was periodic, like the plot in Figure 3 (left), the simulation would likely be stable for a prolonged time. If the plot explodes exponentially or approaches the 
planet's atmosphere (the dashed line on the left-hand plot of Figure 3), then the orbit is considered unstable. Using the mirror's velocity is another way to tell if an orbit is unstable. By comparing the mirror's velocity to its escape velocity (Figure 3, right), we can predict if the mirror will escape its orbit. In this case and others, often the mirror's velocity will overlap the escape velocity, so it can be hard to tell without further information exactly what is going on. If we had the ability to zoom in on a specific part of the plot, it would be easier to see exactly what's going on.
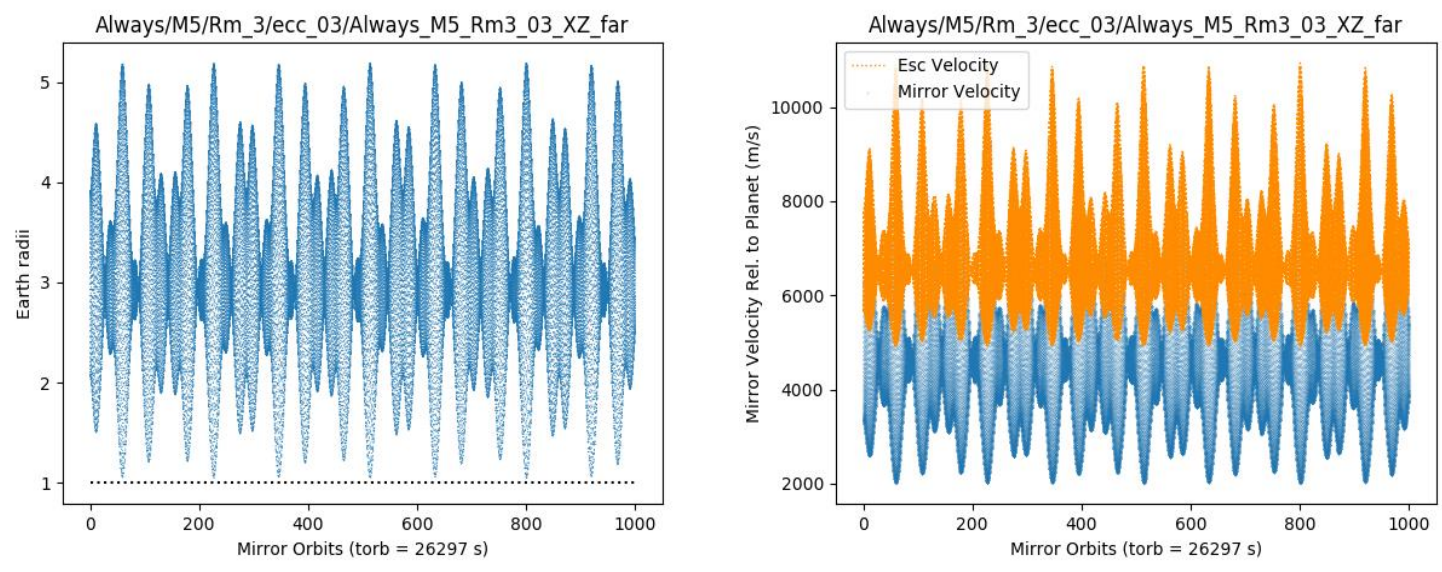

Figure 3: A plot of the mirror's distance to the planet vs simulation time in mirror orbits with the planet's atmosphere represented by the dotted line at the bottom (left) and a plot of the mirror's velocity in blue compared to the escape velocity in orange for the mirror at that point in time (right). In both cases, the mirror is orbiting a planet that's orbiting an M5 star. The mirror is initially placed at the farthest point from the planet, with a semi-major axis of 3 planet radii and an eccentricity of 0.3 , in the XZ plane. For this simulation, radiation pressure is "always on".

The period, eccentricity, and semi-major axis also provide information about the simulation. We can see how the orbit evolves over its lifetime and use that information to further understand how orbits change in the presence of radiation pressure. We also thought it might be of value to plot the changes in the variables described, but so far this information hasn't been useful. Figures 4, 5, and 6 contain this information for the simulation described in Figure 3. 

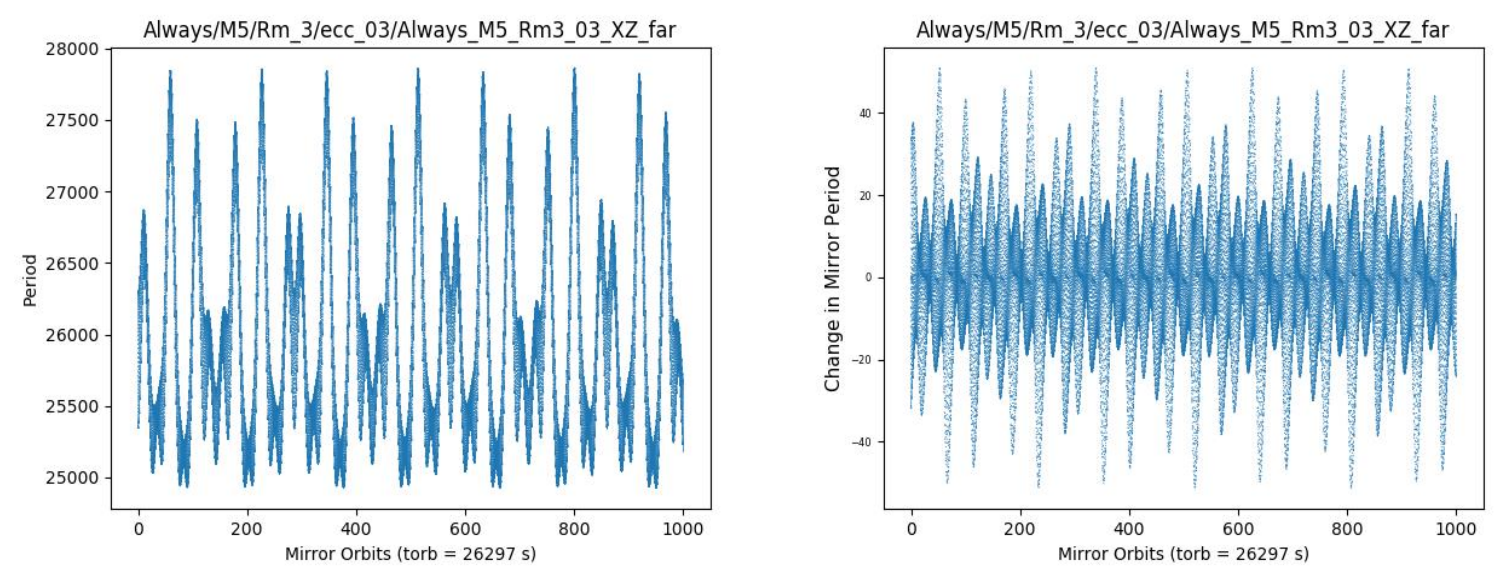

Figure 4: For the same simulation as Figure 3: A plot of the mirror's period vs simulation time in mirror orbits (left) and a plot of the change in the mirror's period vs mirror orbits (right).

As can be seen in figure 4 on the left, the period varies by a little over ten percent of its initial value, with a pattern that seems to repeat. In figure 5, we plotted the eccentricity over time (left), as well as how the eccentricity is changing (right). This orbit varies from nearly circular (when eccentricity is close to 0 ) to quite elliptical, in a pattern that displays multiple periodicities.
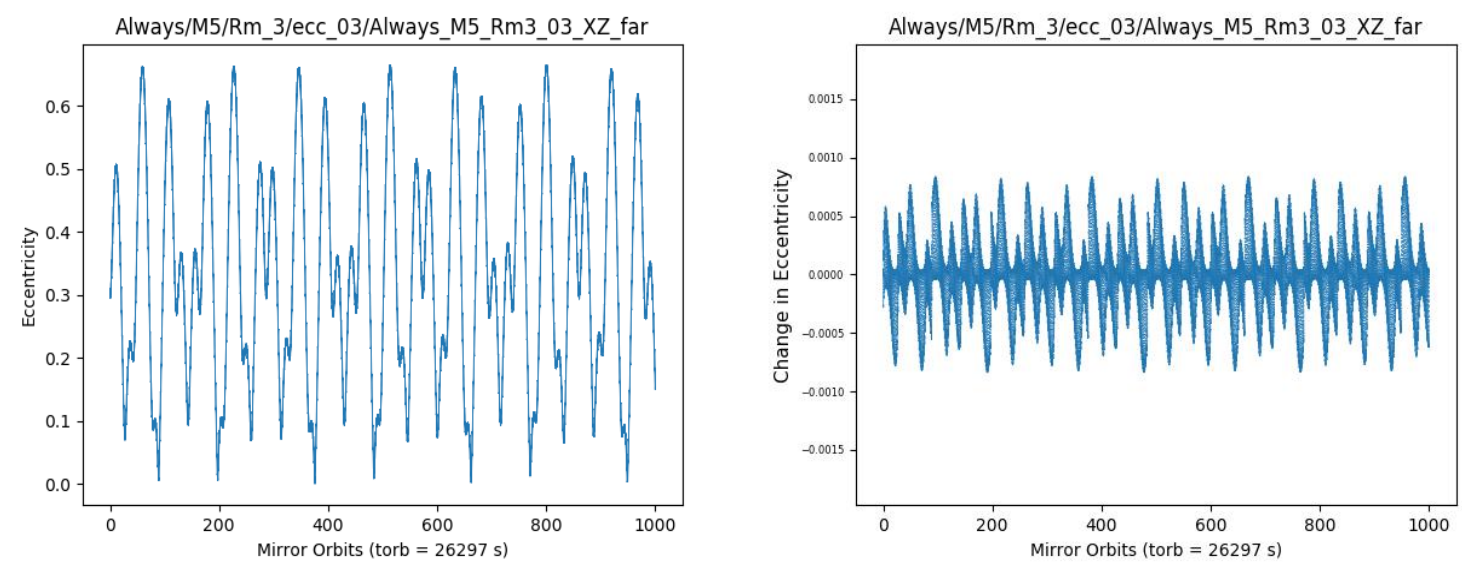

Figure 5: For the same simulation as Figure 3: A plot of the mirror's eccentricity vs simulation time in mirror orbits (left) and a plot of the change in the mirror's eccentricity vs mirror orbits (right).

In figure 6, we graphed the mirror's semi-major axis over time (left). As you can see, this resembles the period plot in figure 4 . This is because the period and semi-major axis are related; the longer the semi-major axis, the bigger the period is. This is shown by Kepler's third law relating the period and semi-major axis: $P^{2}=k a^{3}$, where $P$ is the period, $a$ is the semi-major axis, and $k$ is a proportionality constant. 

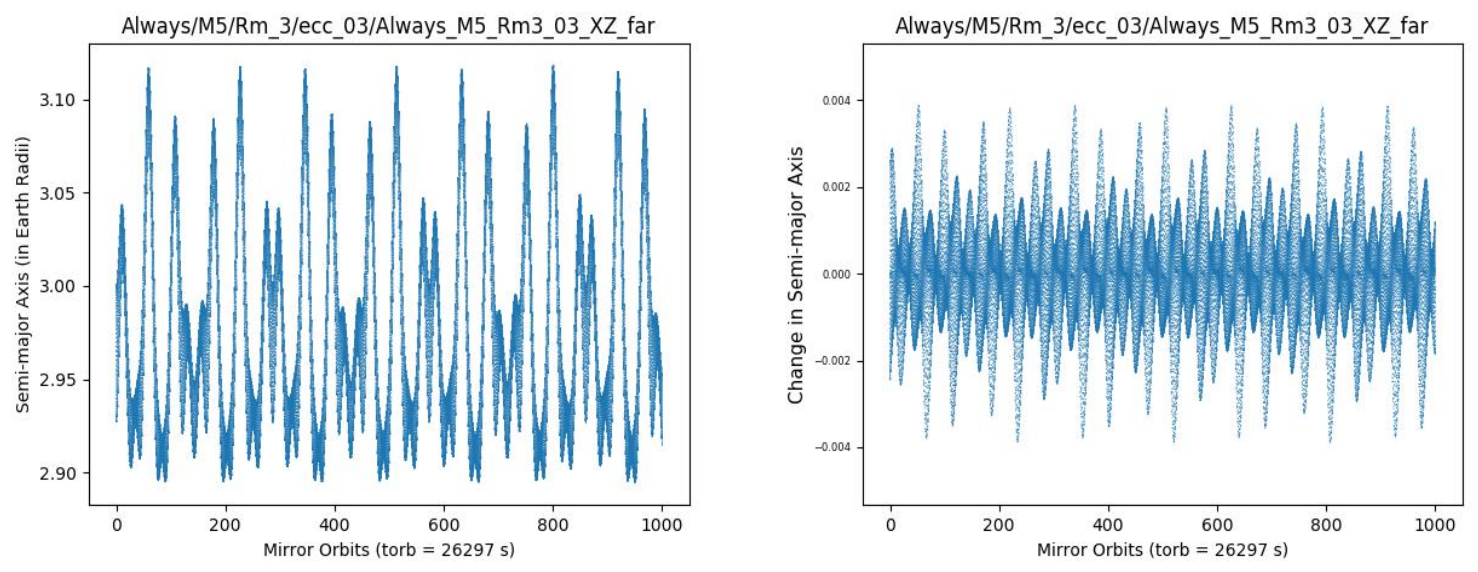

Figure 6: For the same simulation as Figure 3: A plot of the mirror's semi-major axis vs simulation time in mirror orbits (left) and a plot of the change in the mirror's semi-major axis vs mirror orbits (right).

I compiled a list of orbit survival times in seconds as well as in terms of mirror orbits (the time it would take for the mirror to orbit the planet one time if there were no outside influences), along with each simulation's end state (survive, crash, escape). I used the plots that I made to make a list of data trends for each simulation. Analyzing these data led to a list of trends in orbit stability and evolution and helped determine whether surviving orbits are likely to continue to survive.

\section{Discussion:}

Analyzing the data that was output from each simulation and compiling the results revealed that many of the simulations with initial non-zero eccentricity were similar to the equivalent (everything else being equal) initially circular $(e=0)$ simulation. Overall, simulations with smaller initial eccentricities were more similar in result and orbit shape to the equivalent circular simulation than simulations with larger initial eccentricities. Not surprisingly, this suggests that initial orbit shape is typically not the dominant parameter predicting survival time, but that the effect of eccentricity is larger for the least circular orbits.

The RP type affected orbit life as well. In general, for both circular and eccentric simulations, those with no radiation pressure were affected only by gravity and therefore the orbits around mid-sized stars (M0 and K5) were more stable. These were run to more easily compare and understand what happens when radiation pressure or other forces are included in the simulations. If there was only radiation pressure when the mirror was on the far side of the planet (ie Night RP), this led to a generally unstable orbit. If radiation pressure was always “on" (Always RP), the simulations lasted longer. The mirrors had more stable orbits for bigger stars (K0 and G2) and for initial orbit orientations that were perpendicular to the plane of the planet-star system.

However, some eccentric simulations varied greatly from the corresponding circular simulation. In Figure 7, it's shown that the initially circular simulation lasted the requested 1000 orbits, while 
varying the eccentricity even by 0.1 affects the orbit so greatly that the mirror crashes into the planet after only 36 orbits.
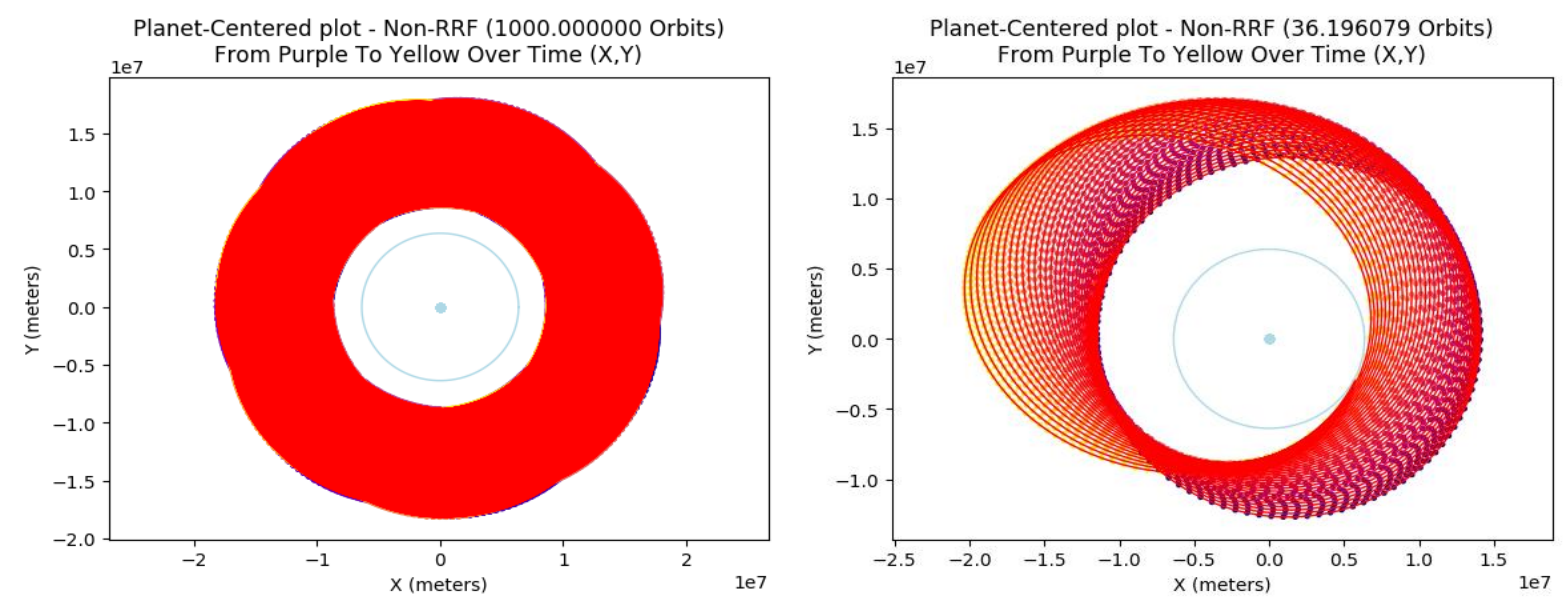

Figure 7: A mirror experiencing Night RP while orbiting a planet around an M5 star at a distance of 2 planet radii. Mirror dot color gradually changes from purple to yellow throughout the simulation, and dots are joined by a red line. The mirror has an initial orientation in the $-\mathrm{XY}$ (clockwise) direction. The planet surface is indicated by the blue circle. The circular simulation (left) lasts for 1000 orbits, whereas the eccentric (e=0.1) simulation (right) crashes after 36 orbits.

In analyzing previous circular simulations, there was a simulation that warranted further investigation. In particular, it was a simulation around an M0 star, set 10 planet radii away, with Night RP, in the $-X Y$ direction. When we decided to try the $-X Y$ orientation, we didn't expect any significant change from the $\mathrm{XY}$ orientation, but it actually resulted in some simulations behaving vastly differently. Then, we ran the 30 simulations that were significantly different from their XY counterparts for 3000 orbits and analyzed their eccentricity and semi-major axis plots, as well as other plots.

One simulation in particular had a unique trend in the evolution of the mirror's distance from the planet. As shown in Figure 8, the plot has "bumpiness" at the top and bottom of the graph. Usually, our distance plots are smooth or they have extreme oscillation, as in figure 3. This simulation had multiple patterns of oscillation overlaid, and the 3000-orbit plot suggested a gradual decrease in the maximum distance of the mirror from the planet, with little to no long-term trend in the minimum mirror-planet distance. 

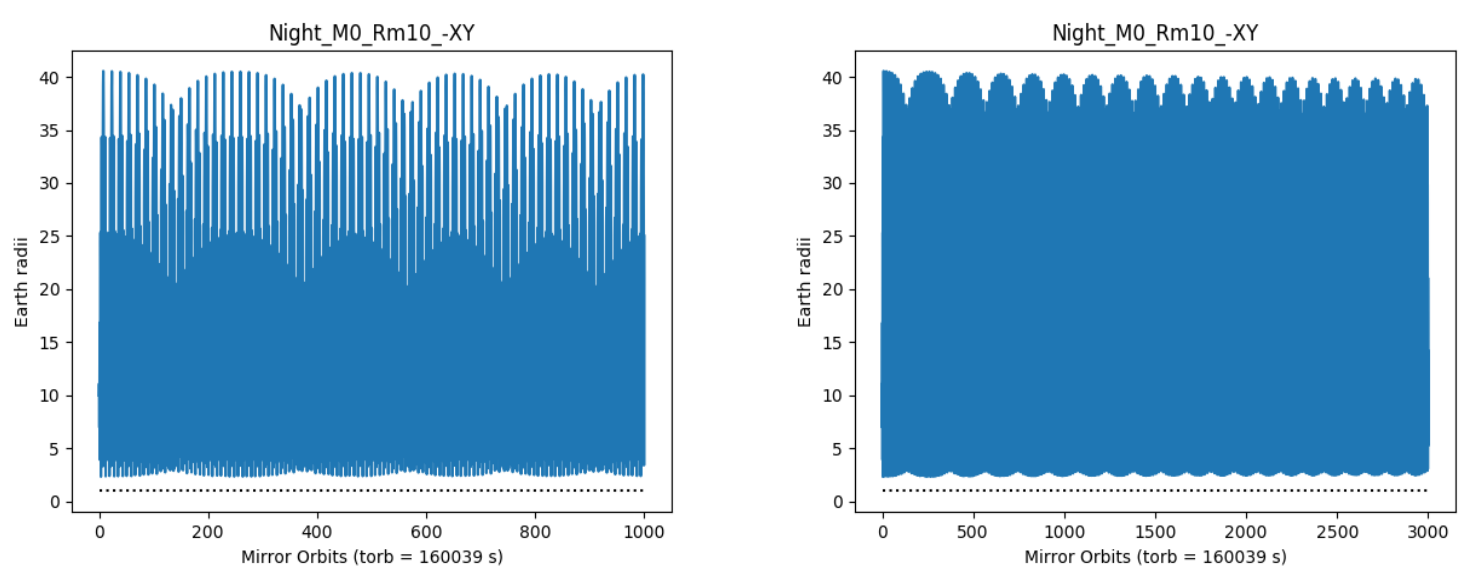

Figure 8: Two plots of the mirror's distance from the planet over time for a mirror initially set 10 planet radii from the planet, orbiting backwards in the XY plane. The planet in this simulation is orbiting an M0 star. Radiation pressure is set to only affect the mirror when it's on the dark side of the planet. Left: 1000 orbits. Right: 3000 orbits.

This simulation was also interesting because the corresponding simulation that orbits forward in the XY plane lasted only 3.27 mirror orbits, as shown in Figure 9. We have not yet analyzed in detail the large discrepancy in such simulations; we are currently working to investigate this. However, in $+X Y$ simulations the mirror and planet orbit are in the same direction, while in the XY simulations they are opposite. In the + XY situation shown in Figure 9, as the mirror first orbits the planet, the planet moves upward in the same direction. Because they are now closer together, the mirror gains kinetic energy. In the $-\mathrm{XY}$ simulation the opposite is occurring. In some of our simulations, this small difference early on causes dramatic changes to the outcome, while in other simulations, the changes are less pronounced.
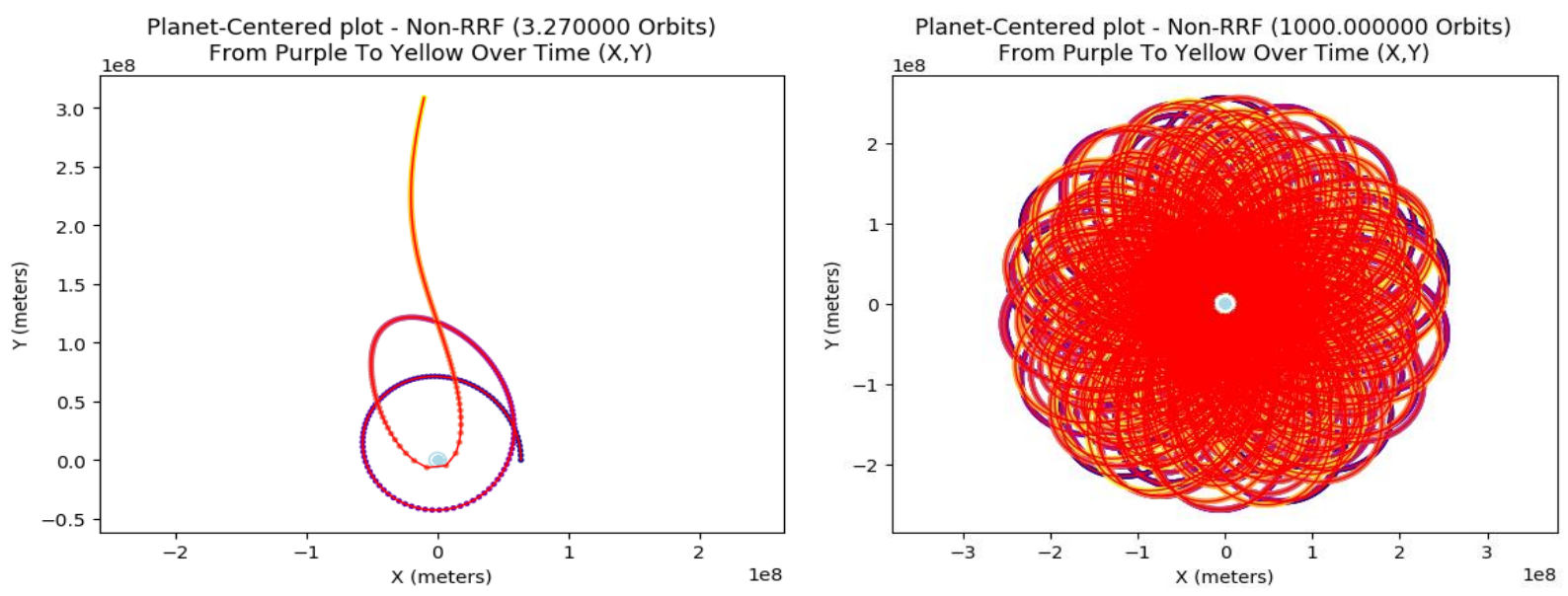

Figure 9: For the simulation described in Figure 8: Two plots showing the path the mirror takes around the planet. (Left) The mirror orbits forward in the XY plane and escapes after 3.27 orbits. (Right) the mirror orbits backwards in the XY plane and lasts for 1000 orbits. 
We wanted to look further into this, so we ran the simulation for 100,000 orbits. To our great surprise, the simulation lasted to completion. Looking again at the mirror's distance from the planet over time in Figure 10, the downward trend in its apocenter continued and became more pronounced.

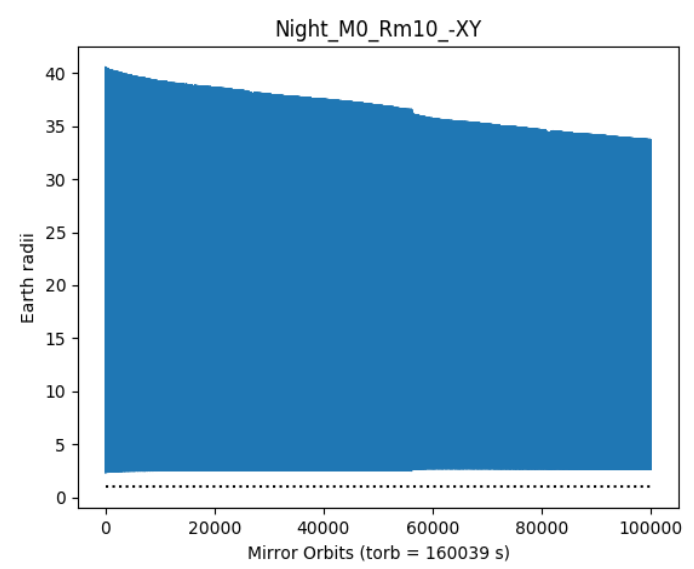

Figure 10: The mirror's distance from the planet over time for a mirror initially set 10 planet radii from the planet, orbiting backwards in the XY plane. The planet in this simulation is orbiting an M0 star. Radiation pressure is set to only affect the mirror when it's on the dark side of the planet. This simulation lasts for 100,000 mirror orbits.

We are currently running this simulation for one million orbits and developing a tool to pause and restart simulations in order to further analyze interim information about simulations that show trends but last for an exceptionally long time.

Future Work:

During 2020-2021, our research team continued to analyze data collected during summer 2020; in particular, we began to compare the results for the same eccentricities at different mirror orbit distances as well as comparing the results for different eccentricities at the same orbit distance. We are still working on a complete analysis as no single factor dominates the survival times. Mirror survival times are affected by (1) the strength of radiation pressure relative to the gravitational influence of both the planet and the star, which are affected by numerous simulation parameters (2) the mirror orbit orientation (which affects the angle at which starlight hits the mirror during its orbit), (3) the version of radiation pressure (Night vs Always), and (4) how far the planet moves around the star during a mirror orbit, since this affects the direction at which starlight approaches the mirror. These factors are all important, with no single factor dominating the trends throughout all simulations.

In the future, we hope to be able to use data trends gathered from our work to determine if an orbit will be stable without running it for an extended period of time. We also would like to use thrusters 
to potentially stabilize an orbit if it looks to be quasi-stable. As mentioned above, it would also be helpful to be able to zoom in on a plot, to see the intricacies of a simulation.

\section{Acknowledgments:}

I'd like to acknowledge Kaisa Crawford-Taylor, who wrote the python package we're currently working with to interface with REBOUND, which can be downloaded freely at http://github.com/hannorein/rebound. I'd also like to acknowledge Dr. Shauna Sallmen, my research advisor, without whom I would be hopelessly lost, as well as UWL's GIS lab manager Steven Fulton, who helps us immensely with improving and fixing the package.

\section{References:}

Korpela, E. J., Sallmen, S. M., and Greene, D. (2015). Modeling Indications of Technology in Planetary Transit Light Curves-Dark-side Illumination. The Astrophysical Journal, 809(2). doi:10.1088/0004-637X/ 809/2/139

Rein, H., and Liu, S. (2012). REBOUND: an open-source multi-purpose N- body code for collisional dynamics. Astronomy and Astrophysics, 537. doi: 10.1051/0004$6361 / 201118085$

Rein, H., and Spiegel, D. S. (2014). Ias15: a fast, adaptive, high-order integrator for gravitational dynamics, accurate to machine precision over a billion orbits. Monthly Notices of the Royal Astronomical Society, 446(2), 1424-1437. doi:10.1093/mnras/stu2164 